\title{
Multi-mass solvers for lattice QCD on GPUs
}

\author{
A. Alexandru, C. Pelissier, B. Gamari, F. Lee \\ Department of Physics, The George Washington University, Washington, DC 20052
}

\begin{abstract}
Graphical Processing Units (GPUs) are more and more frequently used for lattice QCD calculations. Lattice studies often require computing the quark propagators for several masses. These systems can be solved using multishift inverters but these algorithms are memory intensive which limits the size of the problem that can be solved using GPUs. In this paper, we show how to efficiently use a memory-lean single-mass inverter to solve multi-mass problems. We focus on the BiCGstab algorithm for Wilson fermions and show that the single-mass inverter not only requires less memory but also outperforms the multi-shift variant by a factor of two.
\end{abstract}

Keywords: GPU, BiCGstab-M, BiCGstab

\section{Introduction}

While most of the visible matter in the universe is made up of hadrons, particles that experience the strong nuclear force, their structure is still not completely understood. The current understanding is that hadrons are made of quarks that interact via gluons. Quantum chromodynamics (QCD) is the theory that describes their interactions $[1,2]$. The structure of hadrons is determined by a quantum superposition of many quark-gluon configurations: there is no obvious hierarchy among the configurations and the standard perturbative approach is not useful. However, we can use numerical methods to compute hadron properties. These methods are based on a non-perturbative formulation of QCD, lattice QCD [3, 4]: the space-time is approximated by a four dimensional grid, the quarks are viewed as particles hopping between

Email addresses: aalexan@gwu.edu (A. Alexandru), craigp@gwmail.gwu.edu (C. Pelissier), bgamari@physics.umass.edu (B. Gamari), fxlee@gwu.edu (F. Lee) 


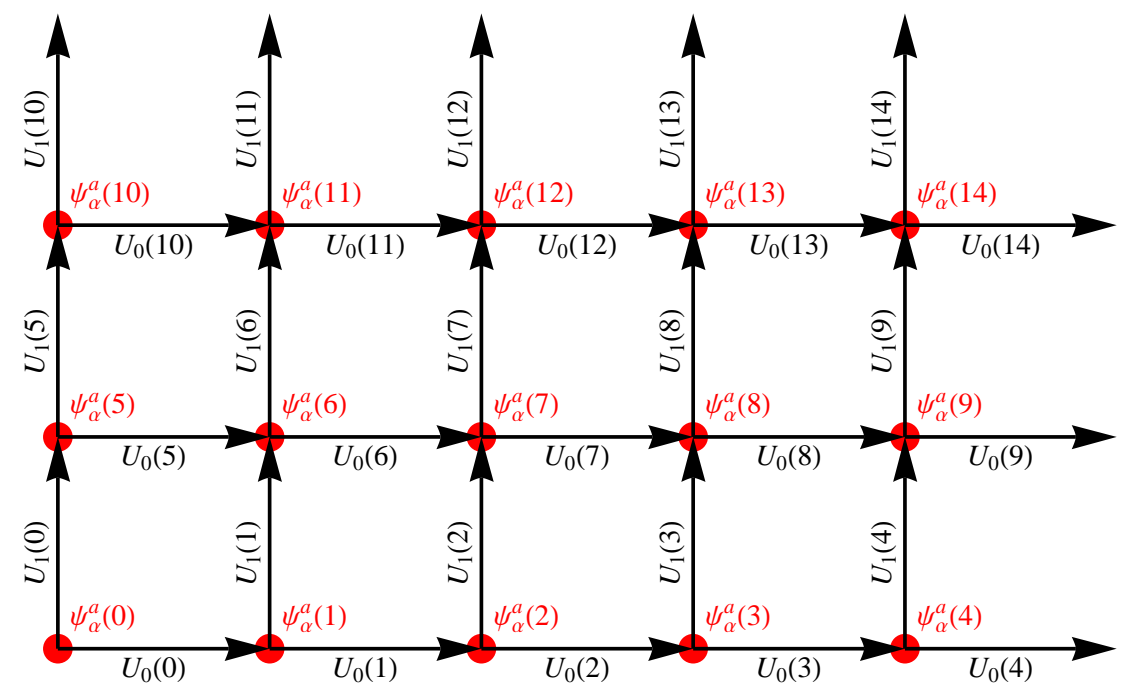

Figure 1: Schematic representation of the lattice discretization: the quark fields $\psi_{\alpha}^{a}$ are associated with the sites of the lattice and the gauge variables $U_{\mu}$ are defined on the links connecting the sites.

the sites of this grid and the gluons are represented by parallel transporters that change the internal state of the quarks as they hop along the given link (see Fig. 1 for a schematic representation). The quantum fluctuations are taken into account by path integral methods in the Euclidean framework.

Formally, the problem is equivalent to a statistical mechanics problem where the observables can be expressed as correlation functions; we use Monte Carlo techniques to estimate these correlations. For definitiveness sake, we write down the equivalent partition function. After rotating to Euclidean times and discretizing the action we get [5]:

$$
Z=\int \mathcal{D} U \mathcal{D} \bar{\psi} \mathcal{D} \psi e^{-S_{g}(U)-\bar{\psi} M(m ; U) \psi} .
$$

The measure $\mathcal{D} U \equiv \prod_{n, \mu} d U_{\mu}(n)$ and $\mathcal{D} \bar{\psi} \mathcal{D} \psi \equiv \prod_{n, a, \alpha} d \bar{\psi}_{\alpha}^{a}(n) d \psi_{\alpha}^{a}(n)$ represents an integral over all degrees of freedom: $U_{\mu}(n)$ is the gauge link at site $n$ and direction $\mu$ which represents the gluon field and $\psi_{\alpha}^{a}(n)$ is the quark field at site $n$ with color index $a$ and spinorial index $\alpha$. The purely gluonic part of the action, $S_{g}(U)$, includes the gluons' kinetic term and the gluon-gluon interaction. The quark contribution is given by $\bar{\psi} M(m ; U) \psi$ where $m$ is the mass of the quark. Since the quark action is quadratic in the quark fields we 
can integrate them out to get

$$
Z=\int \mathcal{D} U e^{-S_{g}(U)} \operatorname{det} M(m ; U) .
$$

The path integral is now expressed completely in terms of the gauge fields $U$, and the quark contribution is given by the determinant of the quark matrix $M(m ; U)$; this matrix is the discretized version of a covariant derivative, and we will discuss its structure later in more detail.

Most lattice QCD calculations are usually separated into two steps: gluonic links generation and correlator measurement. In the first step a set of gauge configurations are generated with a distribution given by the Euclidean action in Eq. 2; each configuration corresponds to a snapshot of the gluonic fields - the quark fields are present in this stage only implicitly by their contribution to the probability measure, $\operatorname{det} M(m ; U)$. In the second stage the hadron correlators are computed for each configuration, and then the ensemble average is calculated; the hadron correlators measure the hadron's ability to propagate through space-time. Their behavior allows us to determine the internal structure of hadrons and their masses. In both stages, the most time consuming part of the calculation relates to manipulating the quark matrix $M(m ; U)$. The typical calculation involves computing $M(m ; U)^{-1} \boldsymbol{b}$ thousands of times for different vectors $\boldsymbol{b}$ and gauge links $U$. The calculation is done iteratively and most of the computation time is spent computing the product between $M(m ; U)$ and intermediate vectors. This is referred to as the fermion-matrix multiplication routine.

Lattice calculations are numerically very expensive: the fermionic matrix is a square matrix with a few million rows. Fortunately, the matrix is very sparse and only a few non-zero elements need to be stored for every row. Since the matrix is sparse and local, the fermion-matrix multiplication routine can be efficiently parallelized; optimized implementations can run double precision calculations at a rate of 1-2 GFlops per processor core on modern CPUs [6]. The numerical requirements for lattice QCD calculations demand the use of large clusters: typically a few hundred to a few thousand processor cores are needed. A new direction in high performance computing has recently become available with the development of general purpose graphic processor units (GPUs). These devices have very good floating point performance, and more importantly for lattice QCD applications, very good memory bandwidth. While these devices are difficult to program, their raw performance is compelling. The advantage of using GPUs for Lattice QCD 
calculations was demonstrated very early [7], and today we have efficient implementations of the fermion-matrix multiplication routine and of the iterative methods that allow us to compute $M(m ; U)^{-1} \boldsymbol{b}[8]$.

Lattice QCD simulations often requires the computation of quark propagators $M\left(m_{i} ; U\right)^{-1} \boldsymbol{b}$ for several quark masses $m_{i}$. This is needed, for example, to study the dependence of physical observables on the quark mass which is required in order to extrapolate to the physical limit (direct simulations are still too expensive). This is also required when we evaluate functions of the quark matrix using rational approximations $[9,10]$. The fermion matrices for different quark masses differ only by a multiple of the identity matrix. They are shifted versions of the same matrix. We can then use multi-shift inverters [11], iterative methods that allow us to compute all quark propagators at once. The fermion-matrix multiplications are only performed once (for the smallest mass) and these results are used to compute all other propagators. The disadvantages of these methods are that they need more memory, require extra linear algebra operations and common acceleration techniques cannot be used.

In order to efficiently use GPUs we need to store most of the data required for computation in the GPU's own memory. This is typically a few gigabytes which is two orders of magnitude less than the memory usually available when running lattice QCD simulations on CPU clusters. Thus, for GPUs we are forced to use single-mass inverters, even when using parallel versions of our codes $[12,13]$. For optimal performance we need to use as few GPUs as possible when parallelizing lattice QCD codes.

In this paper we show how to efficiently solve multi-mass problems for lattice QCD using optimized single-mass inverters. We will focus our attention on the Wilson version of the fermionic matrix [4]. The plan of the paper is the following: in Section 2 we describe the numerical properties of the Wilson fermion matrix, in Section 3 we describe the relevant GPU architectural details and in Section 4 and 5 we describe our GPU implementation of the Wilson-matrix multiplication, the optimizations we used and the challenges we have encountered. In Section 6 we discuss the single-mass and multi-shift versions of the BiCGstab algorithm [14] and the optimization techniques we used. In Section 7 we compare the GPU performance of these two solvers. We find that the solver based on the single-mass inverter, not only uses less memory, but also outperforms the multi-shift inverter by more than a factor of two. 


\section{Wilson fermions}

Lattice QCD simulations spend most of the time in the Wilson $\not D$ routine, a routine that multiplies a lattice vector with the Wilson version of the fermionic matrix [4]. In this section we will describe the Wilson $\not D$ operator, discuss its implementation and present a standard optimization technique.

The Wilson fermionic matrix is a discretized version of the continuum fermionic operator $m+\gamma_{\mu} D_{\mu}$, where $D_{\mu}=\partial_{\mu}+A_{\mu}$ is the covariant derivative associated with the gauge field $A_{\mu}$. For the definition of these operators and other details the reader should consult a lattice field theory textbook (for example [5]) - here we will focus on the numerical aspects of the discretization.

The operator acts on vector functions defined on a four dimensional space. We will only consider the values of these functions at the grid points and we define $\psi_{n}=\psi(n a)$ where $a$ is the lattice spacing and $n$ is a 4 -vector of integers used to index the grid points. The discretized operator, $M(m ; U)$, is then

$$
\begin{aligned}
M(m ; U) \boldsymbol{\psi} & =\left[(m a+4) \mathbb{1}-\frac{1}{2} \not D(U)\right] \boldsymbol{\psi} \\
& =(m a+4) \boldsymbol{\psi}-\frac{1}{2} \sum_{\mu= \pm 1, \ldots, \pm 4} T_{\mu}(U) \boldsymbol{\psi}
\end{aligned}
$$

where $m$ is the quark mass and $\boldsymbol{\psi}$ is the vector associated with $\psi_{n}$ (we will use bold letters to indicate lattice vectors). The fields that describe the quarks are 12 dimensional: they are 4-dimensional spinors with a three dimensional color degree of freedom. $\psi_{n}$ is represented by 12 complex numbers at each site; the 12 complex numbers can be organized as a $3 \times 4$ matrix: $\psi_{n}=\left(\psi_{n}^{c, s}\right)$, with the row index running over colors and the spinorial index over columns. To compute the value of the field $M(m ; U) \boldsymbol{\psi}$ at one particular point we need the value of $\boldsymbol{\psi}$ at the same point and also its value at the neighboring points; in a four dimensional space we have eight neighbors, two in each direction. The values of $\boldsymbol{\psi}$ at the neighboring points are not just copied but rather parallel transported, an operation which we denoted by $T_{\mu}(U)$ :

$$
\begin{array}{ll}
\mu>0: & \left(T_{\mu} \psi\right)_{n}=U_{\mu}(n) \psi_{n+\hat{\mu}}\left(1-\gamma_{\mu}\right) \\
\mu<0: & \left(T_{\mu} \psi\right)_{n}=U_{\mu}(n-\hat{\mu})^{\dagger} \psi_{n-\hat{\mu}}\left(1+\gamma_{\mu}\right),
\end{array}
$$

where $\mu$ represents a direction and $n \pm \hat{\mu}$ represents the forward/backward neighbor of $n$ in the direction $\mu$. The $U$ matrices are $3 \times 3$ special unitary matrices. For every site $n$ on the lattice we have 4 such color matrices, one 


\begin{tabular}{lll}
\hline Direction & vectors \\
\hline$T_{1}^{+}$ & $v_{1}^{\dagger}=\left(\begin{array}{llll}i & 0 & 0 & 1\end{array}\right)$ & $v_{2}^{\dagger}=\left(\begin{array}{llll}0 & i & 1 & 0\end{array}\right)$ \\
\hline$T_{1}^{-}$ & $v_{1}^{\dagger}=\left(\begin{array}{lllll}-i & 0 & 0 & 1\end{array}\right)$ & $v_{2}^{\dagger}=\left(\begin{array}{llll}0 & -i & 1 & 0\end{array}\right)$ \\
\hline$T_{2}^{+}$ & $v_{1}^{\dagger}=\left(\begin{array}{lllll}-1 & 0 & 0 & 1\end{array}\right)$ & $v_{2}^{\dagger}=\left(\begin{array}{llll}0 & 1 & 1 & 0\end{array}\right)$ \\
\hline$T_{2}^{-}$ & $v_{1}^{\dagger}=\left(\begin{array}{lllll}1 & 0 & 0 & 1\end{array}\right)$ & $v_{2}^{\dagger}=\left(\begin{array}{llll}0 & -1 & 1 & 0\end{array}\right)$ \\
\hline$T_{3}^{+}$ & $v_{1}^{\dagger}=\left(\begin{array}{lllll}0 & -i & 0 & 1\end{array}\right)$ & $v_{2}^{\dagger}=\left(\begin{array}{llll}i & 0 & 1 & 0\end{array}\right)$ \\
\hline$T_{3}^{-}$ & $v_{1}^{\dagger}=\left(\begin{array}{lllll}0 & i & 0 & 1\end{array}\right)$ & $v_{2}^{\dagger}=\left(\begin{array}{lllll}-i & 0 & 1 & 0\end{array}\right)$ \\
\hline$T_{4}^{+}$ & $v_{1}^{\dagger}=\left(\begin{array}{lllll}0 & 1 & 0 & 1\end{array}\right)$ & $v_{2}^{\dagger}=\left(\begin{array}{llll}1 & 0 & 1 & 0\end{array}\right)$ \\
\hline$T_{4}^{-}$ & $v_{1}^{\dagger}=\left(\begin{array}{lllll}0 & -1 & 0 & 1\end{array}\right)$ & $v_{2}^{\dagger}=\left(\begin{array}{lllll}-1 & 0 & 1 & 0\end{array}\right)$ \\
\hline
\end{tabular}

Table 1: The vectors needed in the shrink and expand stage for each direction. Since the vectors are column vectors, to save space, we show here the hermitian conjugates which are row vectors.

for each direction. Multiplying the $\psi_{n}$ matrix from the right we have $4 \times 4$ spinor matrices which depend on the direction but not on the lattice position. For $\gamma$-matrices we use:

$$
\gamma_{i}=\left(\begin{array}{cc}
0 & i \sigma_{i} \\
-i \sigma_{i} & 0
\end{array}\right) \quad \text { for } \mathrm{i} \in\{1,2,3\}, \quad \gamma_{4}=\left(\begin{array}{cc}
0 & -1 \\
-1 & 0
\end{array}\right)
$$

where $\sigma_{i}$ are the $2 \times 2$ Pauli matrices.

We stress again that while we have only eight different spinor matrices $\left(1 \pm \gamma_{\mu}\right)$, the color matrices differ from site to site. In fact, any optimized implementation has to take into account the fact that the color matrices need to be transported from memory to the processing unit; their memory layout affects the performance of the code.

The most complex part of the computation is the parallel transport of the neighbors since it involves two matrix multiplications for each direction. The time-consuming part is the color matrix multiplication; the spinor matrix multiplication can be implemented efficiently due to the special form of the spinor matrices. In fact, the spinor multiplication is not computed directly; a standard trick is used that effectively halves the amount of computation needed. The key observation is that the spinorial matrices $\left(1 \pm \gamma_{\mu}\right)$ are projection matrices on a two-dimensional space, i.e. $\left(1 \pm \gamma_{\mu}\right)=v_{1} v_{1}^{\dagger}+v_{2} v_{2}^{\dagger}$, 
where the two vectors depend on direction (see Table 1). We can then reduce the number of color-matrix color-vector multiplications from four to two. The calculation proceeds in three steps:

- Shrinking: In Table 1, for each of the eight direction a pair of 4-vectors is given. We first compute two color vectors: $\psi_{1,2}^{c}=\psi_{n} v_{1,2}$.

- Color multiplication: Each of this vectors are then multiplied with $U$ producing two color vectors $U \psi_{1,2}^{c}$.

- Expansion: the two vectors are combined together to produce the final result:

$$
\psi_{n}^{\prime}=U \psi_{1}^{c} v_{1}^{\dagger}+U \psi_{2}^{c} v_{2}^{\dagger}
$$

It is also worth pointing out that our choice for the $\gamma$-matrices makes the shrinking and expansion steps particularly simple: there are no multiplications, only permutations and additions.

To compute $M(m ; U) \boldsymbol{\psi}$ we need to repeat the steps above for each lattice site. At each site we need to load 9 spinor-vectors, $\psi_{n}$ and $\psi_{n \pm \mu}$, and 8 color matrices, $U_{\mu}(n \pm \mu)$. The final result, $(M(m ; U) \boldsymbol{\psi})_{n}$, has to be stored back in the memory. The total amount of data transported to and from memory is 384 floating point numbers representing 1536/3072 bytes if stored in single/double precision. The calculation at each site requires 1368 floating point operations. The ratio of bytes transported per floating point operation is 1.12 in single precision and 2.25 in double precision.

\section{GPU architecture and programming model}

The methods presented in this paper were tested on NVIDIA GPUs and implemented using the CUDA toolkit [15]. The two architectures that we used for our testing were the older GT200 and the newer Fermi/GF100 architecture. In this section we will describe the relevant features of the GPU architecture and the programming model.

NVIDIA GPUs are designed to support a large number of threads running in parallel. Typically, a GPU has a few hundred computing cores each with one integer and one floating point unit. In single precision, the floating point unit can execute one instruction per clock cycle. Depending on the hardware architecture, the double precision performance is one tenth to one half of the single precision performance. The routines executed on the GPU compute 

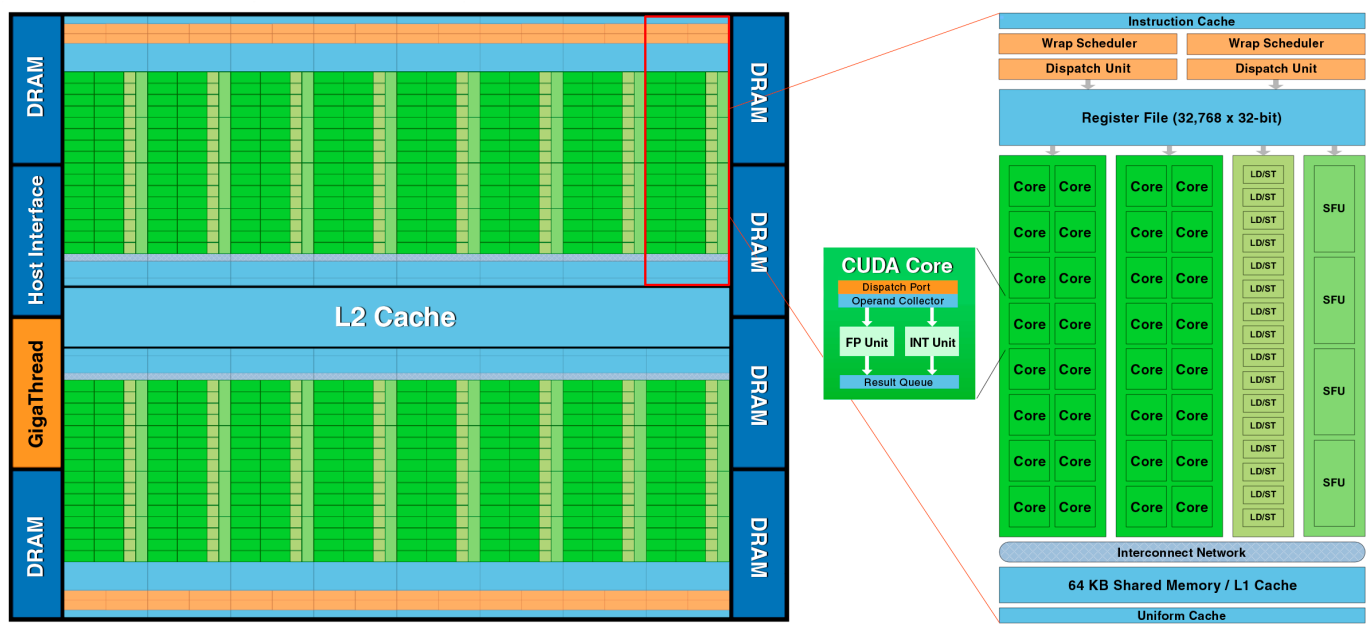

Figure 2: Fermi architecture: 16 streaming multiprocessors (SM) each including 32 CUDA cores, 16 load/store units, 4 special functions units, a large register file, etc. [16]

cores are called kernels and they are issued from the CPU. The CUDA toolkit allows for easy mixing of CPU code and kernels: the GPU code is identified, compiled separately and linked in the final executable automatically.

The GPU architecture has a rich memory model: global device memory, L2 cache (in the case of Fermi), shared memory, etc. The majority of the device's storage is global device memory. This is high-latency off-chip memory with a capacity generally on the order of gigabytes. The CPU memory is accessible from the GPU via the PCI bus which is more than 20 times slower than the GPU's memory bus. In order to efficiently use the GPU, frequently used data has to reside in device memory and transfers to and from CPU memory have to be infrequent. In our codes we store all the required data for the inversion in the device memory and only transfer the result to the CPU memory when the inverter converges.

At the hardware level, the GPU has around a dozen streaming multiprocessors (SM), each with 8-32 compute cores, a large register file and some shared memory. The threads scheduled to run on one SM share the register file and shared memory. To run memory intensive applications efficiently, the GPU has a large bandwidth to the device memory, around $150 \mathrm{~GB} / \mathrm{s}$. However, the device memory latency is a few hundred clock cycles. To hide this latency the SM keeps a pool of active threads that are scheduled to run 
as soon as the required data is loaded from device memory. In order to hide the latency completely, we need a couple of hundred threads to be active for each SM. To quickly switch the active threads in and out of the execution queue, each thread gets exclusive access to its registers as long as the thread is active. Thus, the register file needs to hold the registers for hundreds of threads. This is why the number of registers per thread is limited to 128 for the GT200 architecture and 63 for the Fermi architecture (the number of registers per thread is reduced for Fermi since each SM has four times as many cores whereas the number of registers was only doubled.) When writing kernels we have to be very careful not to exceed this limit, since any additional data will be spilled into device memory resulting in a significant performance loss.

Threads are submitted to the processor divided into groups known as blocks. A basic kernel call

kernel $\ll<$ block_number, block_size $\gg>$ (arguments ...);

will launch block_number $\times$ block_size threads in blocks of size block_size. Simplifying things a little, we think of the kernel call above as launching many threads, each calling an identical function

kernel(arguments ..., thread_number);

where the only difference is the thread_number provided by CUDA runtime. Each block is mapped to a SM which enables cooperation of threads within their block. Threads within a block can quickly share data through their SM's shared memory and synchronize with block-wide barrier instructions. The shared memory can also be used to store temporary data; this is very useful when the number of registers needed for each thread exceeds the hardware limit mentioned above. However, since shared memory size is actually smaller than the register file (for Fermi we have $64 \mathrm{~KB}$ of L1/shared memory vs $128 \mathrm{~KB}$ for the register file), the additional amount of storage is limited. Furthermore, the data has to be carefully laid out in shared memory to avoid bank conflicts (see section 5.3.2.3 of [15]).

Another important architectural detail is that, in order to fully exploit the device memory bandwidth, the memory access needs to be coalesced. This roughly means that threads within a warp, the smallest thread block that gets scheduled for execution (32 for current devices), should access data from a continuous block of memory. To make things clearer it is useful to 

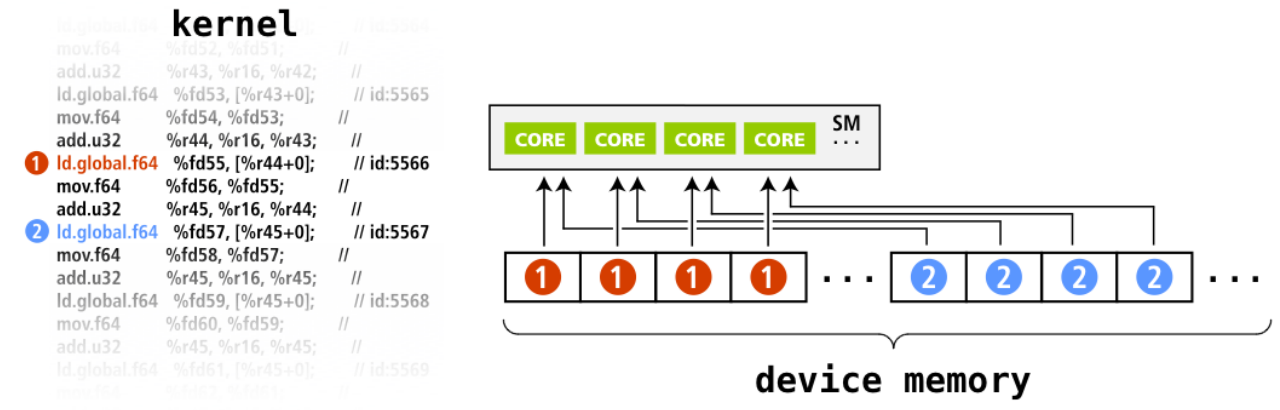

Figure 3: Computing model: the kernel is a set of instructions executed in parallel on all computing cores. When a load/store instruction is encountered, all cores request a chunk of data from memory (usually register size). For best performance, these chunks should form a contiguous memory block.

discuss the programing model we used to design our codes. While the GPU programming model is more flexible, we find it useful to model the GPU as a SIMD ${ }^{1}$ device: each computing core executes the same set of instructions on a different piece of data. We assume that each thread operates independently, all threads execute the same set of instructions and each thread loads and stores the input and output based on its thread index. To ensure that the memory access is coalesced, we arrange the data loaded by the same kernel instruction in a contiguous array (see Fig. 3). Thus the natural layout for our data is a structure of arrays rather than an array of structures. For example an array of complex numbers will be stored as an array with all real parts first and another array with the imaginary parts.

To sum up, in order to extract maximum performance from our kernels, we need to store all relevant data in device memory, arrange it such that the GPU cores that operate in lock-step load/store data in continuos blocks and design kernels that use as few register as possible so that we can schedule a large number of active threads to hide the latency of the device memory.

\footnotetext{
${ }^{1}$ SIMD stands for single instruction, multiple data.
} 


\section{Implementation details}

In this section we discuss our GPU implementation of the Wilson matrix multiplication routine. We discuss our implementation strategy, the data layout used for our vectors and gauge links and present the multiplication kernel in detail paying close attention to the register usage. We conclude this section with a discussion of the performance of the single and double precision kernels showing that they nearly saturate the bandwidth limits.

For lattice QCD simulations, most of the computation time is spent in the routine that implements vector-matrix multiplication for the fermionic matrix. Consequently, our efforts focused on optimizing the implementation of this routine. This routine is well suited for parallel programming since the steps described in Eqs. 3, 4 have to performed independently for every site in the lattice. Moreover, each of the eight parallel transporters described in Eq. 4 can be performed independently.

The most important design decision was the degree of thread granularity. On one hand, it is preferable to divide the computation in as many pieces as possible. For example, we can have each thread compute only one parallel transporter for one site. In this case, the threads would be light-weight and we can keep them active in sufficient numbers to hide memory latency. On the other hand, when a thread is responsible for a larger fraction of the computation, the data is used more efficiently. For example, if each thread computes the matrix-vector multiplication for sites that belong to a $2^{4}$ tile, then the amount of data that needs to be moved per site is reduced to $65 \%$ of the data moved when computing one site per thread. Unfortunately, the register requirement per thread increases roughly 16 times. A possible solution would be to use shared memory to load in the $2^{4}$ tile but this solutions adds considerable complexity to the code. We decided that the optimal solution is to have each thread responsible for one lattice site.

The layout of our data structures had to be carefully chosen to achieve maximum performance. The vectors have the following structure:

$$
\begin{aligned}
\boldsymbol{\psi} & =[\overbrace{\operatorname{color}_{0}, \operatorname{color}_{1}, \operatorname{color}_{2}}^{\text {real part }} \overbrace{\text { color }_{0}, \text { color }_{1}, \text { color }_{2}}^{\text {imaginary part }}] \\
\operatorname{color}_{i} & =\left[\operatorname{spin}_{0}, \operatorname{spin}_{1}, \operatorname{spin}_{2}, \operatorname{spin}_{3}\right] \\
\operatorname{spin}_{i} & =\left[\operatorname{site}_{0}, \operatorname{site}_{1}, \ldots, \operatorname{site}_{N}\right]
\end{aligned}
$$

where $N$ is the number of sites on the lattice. We have then $2 \times 3 \times 4=24$ arrays, each of length $N$. The gauge links are represented by four $3 \times 3$ 
matrices for each site. Their layout is similar to the one used for vectors:

$$
\begin{aligned}
U & =[\overbrace{\operatorname{dir}_{x}, \operatorname{dir}_{y}, \operatorname{dir}_{z}, \operatorname{dir}_{t}}^{\text {real part }}, \overbrace{\operatorname{dir}_{x}, \operatorname{dir}_{y}, \operatorname{dir}_{z}, \operatorname{dir}_{t}}^{\text {imaginary part }}] \\
\operatorname{dir}_{i} & =\left[\operatorname{row}_{0}, \operatorname{row}_{1}, \operatorname{row}_{2}\right] \\
\operatorname{row}_{i} & =\left[\operatorname{column}_{0}, \operatorname{column}_{1}, \operatorname{column}_{2}\right] \\
\operatorname{column}_{i} & =\left[\operatorname{site}_{0}, \operatorname{site}_{1}, \ldots, \operatorname{site}_{N}\right]
\end{aligned}
$$

As we discussed in the previous section, the data is arranged as a structure of arrays. The particular order of these lattice-size arrays has little effect on the performance; we could switch the spin and color indices within the vector layout without penalty. On the other hand, if we rearrange the data so that the threads access it with a stride the performance can easily decrease by an order of magnitude.

The way the lattice sites are mapped in these linear arrays is not specified and, as long as the choice is made consistently, it can be tailored to our needs. For example, to implement even-odd preconditioning we choose a map that stores first the even parity sites and then the odd parity ones. The matrixvector multiplication routine uses this map to compute the position of the neighbors in this linear array. To keep the routine flexible we can either use layout routines to compute these indices on the fly, or precompute the offsets of the neighbors and store them in eight arrays. We found that precomputing the neighbors' offsets is the best strategy; it only adds $8 \times 4=32$ bytes to the data that needs to be transported per site which is a small overhead.

A schematic presentation of the matrix-vector multiplication routine is presented in Algorithm 1. The subroutines SHRINK, COLOR-MULT and EXPAND are straightforward implementations of the steps described in Section 2. It is important to note that, at a minimum, the kernel needs space to store dest, spinor and half, 60 numbers requiring 60/120 32-bit registers when using single/double precision. When using double precision this poses a challenge. For the older GT200 architecture where the limit of 32-bit register per thread is 128 , the compiled kernel can almost fit completely in registers with very few temporaries stored in device memory. For the newer GF100 architecture the register limit is 63 and there are too many temporaries stored in device memory - the performance is reduced significantly. To work around this problem, we store dest in shared memory and spinor and half in the register file.

The performance of the DSLASH routine as a function of the lattice size 


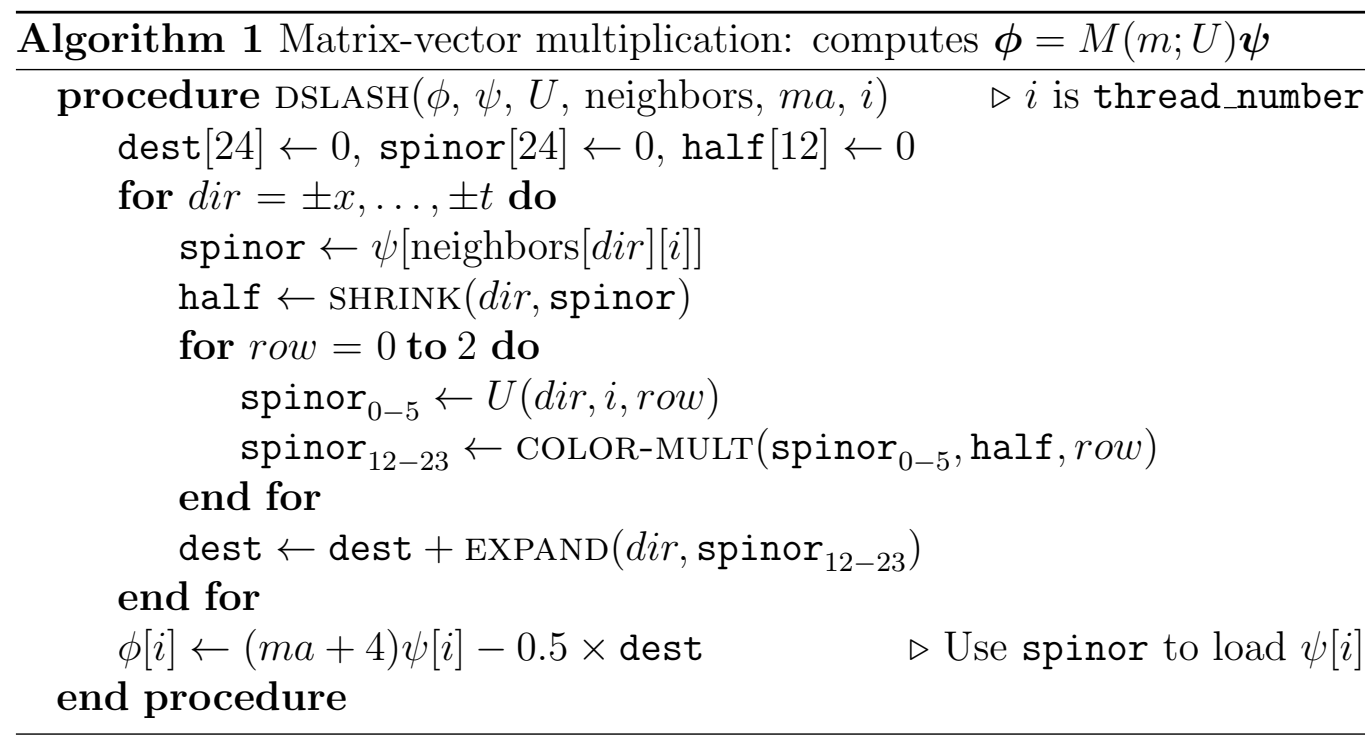

is presented in Fig. 4. We see that for lattice sizes larger than $16^{4}$ performance saturates slightly above 50 GFlops for double precision kernels and around 130 GFlops for single precision. The performance of our kernels is competitive with the performance of QUDA library [8], especially the double precision kernel. To gauge the efficiency of our implementation, it is instructive to compare its performance with the maximum allowed by the memory bandwidth. For GTX 480 the theoretical bandwidth is $177.7 \mathrm{~GB} / \mathrm{s}$, however this is not a very useful number since it is not clear whether this bound can actually be achieved. A more useful bound is to take the peak bandwidth actually achieved by a very simple kernel, a vector addition routine. This routine is perfectly parallel, its register requirement is minimal and the memory access is completely sequential. As seen from Fig. 5, the maximal bandwidth for this simple routine is about $145 \mathrm{~GB} / \mathrm{s}$. In Section 2 we calculated the computational density for the matrix-vector multiplication routine to be 1.12 bytes/flop in single precision and 2.25 in double precision. To reach a bandwidth of $145 \mathrm{~GB} / \mathrm{s}$ the performance of the kernels needs to be 129.5 GFlops in single precision and 65 GFlops in double precision. It is easy to see from Fig. 4 that the single precision kernel saturates this bound while the double precision kernel achieves $80 \%$ of this limit. The most likely reason is the fact that the maximum number of active threads that we can schedule for this kernel is not sufficient to hide the memory bus latency.

We find that given the strategy we used, our matrix-vector multiplication 


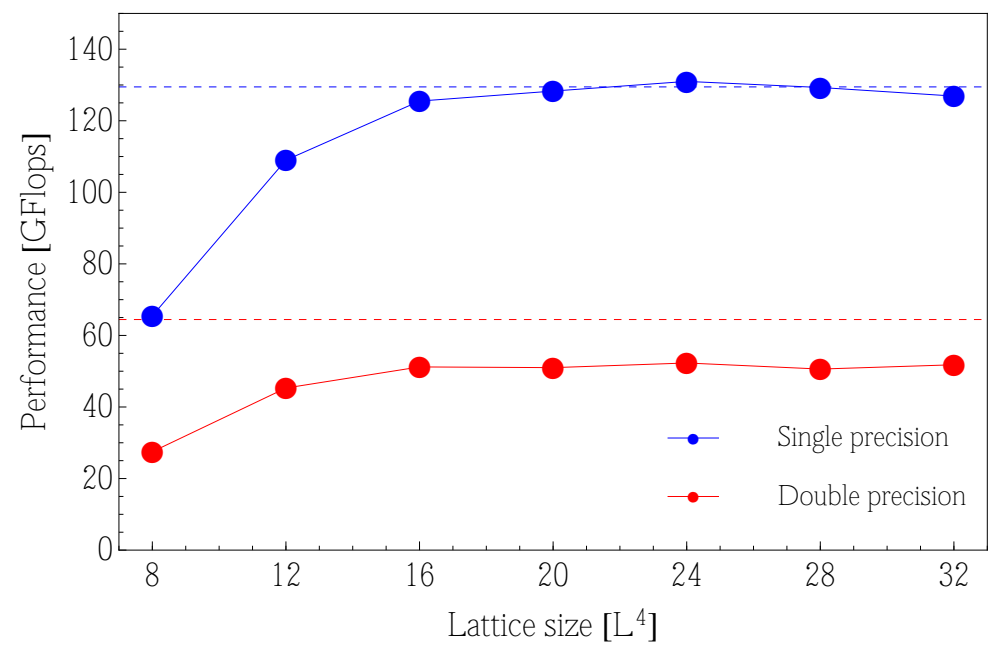

Figure 4: DSLASH routine performance on GTX 480 as a function of the lattice size. The dashed lines represent performance bounds imposed by bandwidth limits (see text for details).

kernels are implemented nearly optimally. To improve their performance, we would need to decrease the amount of data that needs to be moved from the device memory to the GPU. This can be accomplished either by using a caching strategy or by employing a number of techniques to reduce the bandwidth: gauge fixing in the temporal direction, using a compressed representation for the $S U(3)$ matrices, using a different set of $\gamma$-matrices. The bandwidth-saving strategy was employed by QUDA [8]. For the single precision kernels, it was found that each of these techniques improves the performance by about $10 \%$ resulting in a significant improvement when used together. For double precision kernels the gains were more modest. The only downside is that the code becomes considerably more complex. We decided to keep our kernels simpler to make the transition to a multi-GPU framework smoother [13].

\section{Vector utilities}

To implement our inverters, in addition to the matrix-vector multiplication routines we need to implement utilities for vector operations: vector addition, multiplication with real and complex scalars, scalar product and 
norm, etc. These routines are bandwidth limited since their computation density is very low. The kernels are light-weight and simple to implement. To judge the implementation efficiency it is more instructive to look at bandwidth utilization rather than floating point performance.

The problem with these kernels is that very often we need a combination of vector operations. For example, when we want to evaluate $\boldsymbol{\psi}_{3} \leftarrow \boldsymbol{\psi}_{1}+\alpha \boldsymbol{\psi}_{2}$ we can either break the computation in two steps, a vector multiplication and an addition, or write a new kernel. In the first scenario the performance is suboptimal since a temporary vector, $\alpha \boldsymbol{\psi}_{2}$, is transported from the GPU to device memory and back. Since these kernels are bandwidth bound this severely impacts the code performance. The other solution is to write a kernel for every linear operation, or at least for the ones used most frequently. The problem with this approach is that it is error prone and very inflexible. When we change the vector layout all these kernels have to be re-written.

To solve this problem we decided to use expression template techniques to generate the required kernels on the fly [17]. This technique uses the generic programing features offered by templates in $\mathrm{C}++$ to generate kernels at compile-time. The advantage is that we can write our algorithms using clear mathematical syntax, the kernels are generated only when they are needed and the code generated is optimal. The disadvantages are that the compilation time increases and the code is brittle: a slight error causes many pages of errors that offer little information as to what caused the compilation error.

Our expression template implementation follows closely established techniques [17]. Two details worth mentioning are the iterators used and how we dealt with complex numbers. In order to implement expression templates for GPU vectors we needed a special class of iterators; these were provided by the Thrust library [18]. As we pointed out in the beginning, most vector operations traverse the arrays sequentially. However, multiplication with complex scalars is special: the real and imaginary parts of the numbers at a given offset are needed. As discussed in the previous section, the layout of the GPU vectors stores the real and imaginary parts in separate arrays. To present a unified view of these arrays to our expression template implementation, we always represent GPU arrays of complex numbers with the real part stored in the first half of the array and the imaginary part in the second half; this is the reason for having the real/imaginary index as the fastest varying index in the layouts described in Eq. 7 and Eq. 8.

In Fig. 5 we present the performance of two kernels generated by the 


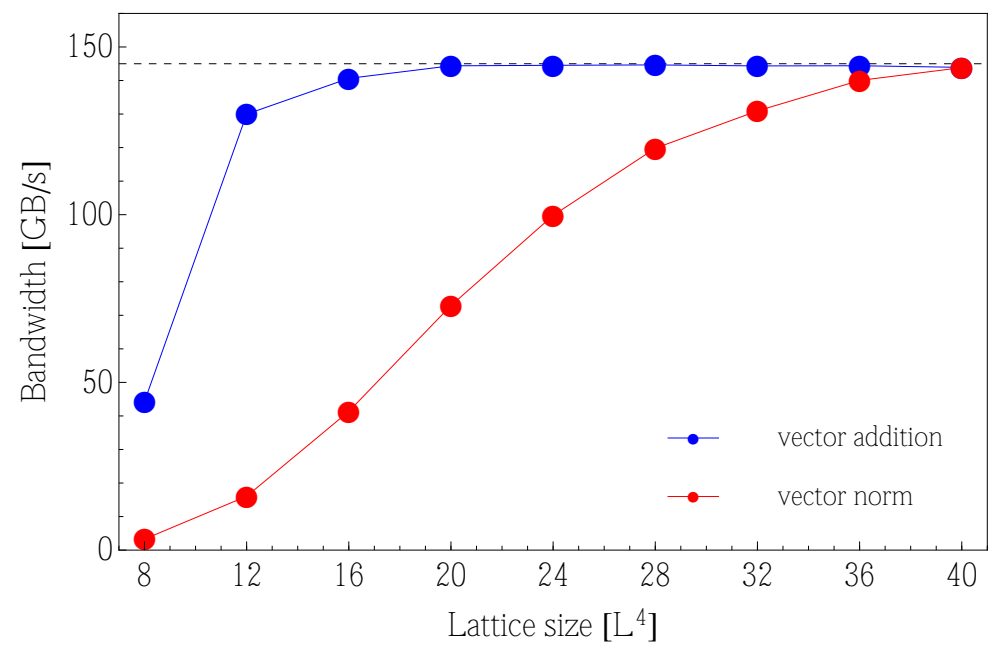

Figure 5: Vector routine performance on GTX 480 as a function of the lattice size. These routines are bandwidth bound and it is more informative to plot their bandwidth rather than floating point performance.

compiler, the vector addition kernel and the norm calculation kernel. The vector addition kernel performance is representative of all sequential access kernels; in particular, we measured the bandwidth for $\boldsymbol{\psi}_{3} \leftarrow \alpha \boldsymbol{\psi}_{1}+\beta \boldsymbol{\psi}_{2}$ with $\alpha, \beta$ being real, complex or zero. The bandwidth is similar for all these operations. On the other hand, calculations of vector norm and scalar products are different. The data needs to be reduced, and this involves communication which slows the kernel down affecting performance. We see that for lattices larger than $16^{4}$ the sequential kernels are saturating the device's memory bandwidth, whereas the reducing kernels need much larger vectors to run optimally. It is important to notice that these very simple kernels peak at about $145 \mathrm{~GB} / \mathrm{s}$ bandwidth - since they cannot be compute-bound and they are extremely light-weight, we think that this offers a good measure of the GPU's maximal bandwidth. Before we conclude, we want to mention that one of the steps in the BiCGstab algorithm, $\boldsymbol{\psi} \leftarrow \boldsymbol{\psi}-\beta \boldsymbol{s}+\chi \boldsymbol{w}$, is different in structure from the kernels tested above. We measured the performance of the automatically generated kernel for this step and we find it to be optimal (similar to the other sequential kernels), as expected. 


\section{Multi-mass solvers}

As mentioned in Section 1, lattice calculations often require the solutions to linear systems

$$
M\left(m_{i} ; U\right) \boldsymbol{\psi}_{i}=\boldsymbol{b}
$$

for several quark masses $m_{i}$. We will refer to such a set of linear equations as a multi-mass system and any method to obtain the corresponding solutions as a multi-mass solver.

For practical reasons these systems are solved using iterative methods. A common choice is the so called Krylov inverters which iteratively build up the associated Krylov space, $\mathcal{K}_{n}(A, \boldsymbol{v})$, starting with some vector $\boldsymbol{v}$

$$
\mathcal{K}_{n}(A, \boldsymbol{v}) \equiv \operatorname{span}\left\{\boldsymbol{v}, A \boldsymbol{v}, A^{2} \boldsymbol{v}, \ldots, A^{n-1} \boldsymbol{v}\right\}
$$

and seek the solution in this space by minimizing the residual [19, 11]. These inverters can be used to determine the solution to Eq. 9 for a given quark mass. We refer to these methods as single-mass inverters. Solutions to multimass systems can then be obtained by using the single-mass inverter for each mass separately.

There is another class of Krylov solvers designed to efficiently invert a set of shifted-linear systems, i.e. a set of systems of the form

$$
A\left(\sigma_{i}\right) \boldsymbol{x}_{i}=\boldsymbol{b} \quad \text { where } \quad A\left(\sigma_{i}\right)=A+\sigma_{i} \mathbb{1},
$$

and $\sigma_{i}$ 's are referred to as the shifts. The advantage of multi-shift inverters is that they use fewer matrix-vector multiplications compared to inverting each system individually. This is achieved by building only the Krylov space $\mathcal{K}_{n}(A, v)$ associated with the unshifted solution. The Krylov spaces for the shifted problems, $\mathcal{K}_{n}\left(A\left(\sigma_{i}\right), \boldsymbol{v}\right)$, are identical. For systems where the matrixvector multiplication is significantly more expensive than vector operations, these methods can be very effective. From Eq. 3, it is clear that $M\left(m_{i}, U\right)$ can be cast in this form where each quark mass $m_{i}$ is associated with a shift $\sigma_{i}$. These methods are then well-suited for solving multi-mass problems. We will refer to methods of this type as multi-shift inverters.

One disadvantage of multi-shift inverters is that they do not lend themselves to certain optimizations available to single-mass inverters. Moreover, they require for $n$ shifts the storage of $2 n+1$ additional vectors [11]. Due to the current memory constraints on GPUs the extra storage required for the multi-shift inverters can be highly constraining. This constraint is also 
present in multi-node systems due to limited scalability. As a result, an optimized solver based on a single-mass inverter would be ideal, provided there is no substantial performance loss. This section is organized as follows: in Section 6.1 we review some relevant details for multi-shift inverters. In Section 6.2 we discuss optimization techniques applicable to single-mass inverters: initial guess tuning, even-odd preconditioning and mixed-precision solvers.

\subsection{Multi-shift inverter}

The convergence rate for an iterative inverter is determined by the conditioning number of the matrix. Systems with larger conditioning numbers will in general converge less quickly. For multi-shift inverters it is important to choose the unshifted matrix to have the largest conditioning number. Since the convergence is determined by the solution of the unshifted system, a different choice would cause the inverter to exit prematurely.

For the fermionic matrix, the conditioning number is inversely proportional to the quark mass. Therefore, systems with smaller quark masses will converge more slowly. We will abuse the notion of singularity slightly by referring to systems with smaller quark masses as being more singular. In setting up the shifted systems we take the unshifted system to be the most singular system which we denote by $M\left(m_{s} ; U\right)$ where $m_{s}=\min \left(m_{i}\right){ }^{2}$ We then define the shifts $\sigma_{i}$ such that

$$
M\left(m_{i} ; U\right)=M\left(m_{s} ; U\right)+\sigma_{i} \mathbb{1} \quad m_{i} \neq m_{s}
$$

For $N$ quark masses we will have $N-1$ shifts. The multi-shift inverter then returns the solutions to

$$
\left[M\left(m_{s} ; U\right)+\sigma_{i} \mathbb{1}\right] \boldsymbol{\psi}_{i}=\boldsymbol{b}
$$

for all $\sigma_{i}$ as well as the unshifted solution $\boldsymbol{\psi}_{0}$. The inverter stops when the residual of the unshifted solution,

$$
\boldsymbol{r} \equiv \boldsymbol{b}-M\left(m_{s} ; U\right) \boldsymbol{\psi}_{0}
$$

satisfies the exit criterium $\|\boldsymbol{r}\|<\epsilon\|\boldsymbol{b}\|$ where $\epsilon$ is the desired accuracy. Since we set the unshifted matrix, $M\left(m_{s} ; U\right)$, to the value of the most singular

\footnotetext{
${ }^{2}$ In the presence of interactions zero quark mass is actually achieved for $m=m_{c}<0$. The most singular mass is the one closest to $m_{c}$.
} 
mass, this exit criterion guarantees the algorithm, in exact arithmetic, will not exit before all solutions converge to the desired accuracy. Lastly, we note that less singular systems will converge more quickly, and it is therefore advantageous to stop updating them once they have converged. This can be achieved at virtually zero cost and is done in our implementation.

\subsection{Optimized single-mass inverter}

In this section, we discuss the implementation of an optimized single-mass inverter as a multi-mass solver. The single-mass based solver we implemented for this study utilizes even-odd preconditioning as well as mixed precision, neither of which can be utilized by multi-shift inverters. A third advantage of a single-mass inverter is the ability to make an initial guess for the solution. In this study we investigate two strategies for making initial guesses. One strategy relies on a polynomial extrapolation, and the other minimizes the residual in the space spanned by the solutions obtained for previous mass values.

\subsubsection{Polynomial extrapolation}

In the polynomial extrapolation method, one uses the previous solutions to construct a polynomial from which the solution to the subsequent mass can be estimated by extrapolation. The estimated solution is then used as an initial guess. If the true function which maps $m$ values to their solutions is sufficiently smooth and does not vary rapidly over the range being considered, this method is expected to generate a good guess. Specifically, suppose you have a set of values $m_{1}, m_{2}, \ldots, m_{N}$ ordered such that $m_{1}<m_{2}<\cdots<m_{N}$ and a set of previously determined solutions $\boldsymbol{\psi}_{1}, \boldsymbol{\psi}_{2}, \ldots, \boldsymbol{\psi}_{k}$. From the set of solutions, one can construct the polynomial

$$
\boldsymbol{\psi}(m)=\boldsymbol{c}_{0}+\boldsymbol{c}_{1} m+\boldsymbol{c}_{2} m^{2}+\cdots+\boldsymbol{c}_{k} m^{k}
$$

with the coefficients $\boldsymbol{c}_{0}, \ldots, \boldsymbol{c}_{k}$ chosen such that $\boldsymbol{\psi}\left(m_{i}\right)=\boldsymbol{\psi}_{i}$ for $i=1, \ldots, k$. We use this polynomial to make an initial guess $\boldsymbol{\psi}\left(m_{k+1}\right)$ for the solution $\boldsymbol{\psi}_{k+1}$. To determine the coefficients, one must invert the associated Vandermonde's matrix

$$
\left[\begin{array}{cccc}
1 & m_{1} & m_{1}^{2} & \\
1 & m_{2} & m_{2}^{2} & \cdots \\
1 & m_{3} & m_{3}^{2} & \\
& \vdots & &
\end{array}\right]\left[\begin{array}{c}
\boldsymbol{c}_{0} \\
\boldsymbol{c}_{1} \\
\boldsymbol{c}_{2} \\
\vdots
\end{array}\right]=\left[\begin{array}{c}
\boldsymbol{\psi}_{1} \\
\boldsymbol{\psi}_{2} \\
\boldsymbol{\psi}_{3} \\
\vdots
\end{array}\right]
$$


The solution of such a linear system is well known, and is given by

$$
\boldsymbol{\psi}(m)=\sum_{j} \boldsymbol{\psi}_{j} \prod_{i=1, i \neq j}^{k} \frac{m-m_{i}}{m_{j}-m_{i}}
$$

\subsubsection{Solution space minimization}

The second method considered is solution space minimization. As suggested by the name, this method minimizes the 2-norm of the residual in the space spanned by the solutions thus far obtained. This method is motivated by the Krylov inverters which try to minimize the residual in the Krylov space. Instead of the Krylov space, we use the space spanned by the solutions obtained for previous masses. This minimization process is desirable since it can be achieved without any additional matrix vector multiplication. Its overall cost in practice is essentially zero.

To minimize the residual $\boldsymbol{r}$, one considers a general superposition of the previously determined solutions $\boldsymbol{\psi}_{1}, \boldsymbol{\psi}_{2}, \ldots, \boldsymbol{\psi}_{m}$ viz.

$$
\boldsymbol{v}(\vec{c})=\sum_{j} c_{j} \boldsymbol{\psi}_{j}
$$

When minimizing $\|\boldsymbol{r}\|_{2}$ with respect to $\vec{c}$, one finds

$$
\sum_{j} c_{j} \boldsymbol{d}_{i}^{\dagger} \boldsymbol{d}_{j}=\boldsymbol{d}_{i}^{\dagger} \boldsymbol{b}
$$

where

$$
\boldsymbol{d}_{k}=\boldsymbol{b}+\left(m-m_{k}\right) a \boldsymbol{\psi}_{k},
$$

and $m$ is the mass for which the solution is to be determined. The coefficients $c_{j}$ can then be determined readily using standard numerical methods.

\subsubsection{Even-odd preconditioning}

Even-odd preconditioning of the fermionic matrix speeds up the convergence of the inverter by a factor of 2 to 3 . The even-odd preconditioning follows from first noticing that the fermionic matrix can be written as

$$
M(m ; U)=(m a+4) \mathbb{1}-\frac{1}{2}\left(\begin{array}{cc}
0 & \not D_{e o}(U) \\
\not D_{o e}(U) & 0
\end{array}\right)
$$

when we separate the even and odd parity sites (the parity of a site $n$ is the parity of $n_{x}+n_{y}+n_{z}+n_{t}$ ). In the following, we suppress the explicit gauge 
field dependence. The linear system which needs to be solved can then be written as

$$
\left[(m a+4) \mathbb{1}-\frac{1}{2}\left(\begin{array}{cc}
0 & \not D_{e o} \\
\not D_{o e} & 0
\end{array}\right)\right]\left(\begin{array}{l}
\boldsymbol{\psi}_{e} \\
\boldsymbol{\psi}_{o}
\end{array}\right)=\left(\begin{array}{c}
\boldsymbol{b}_{e} \\
\boldsymbol{b}_{o}
\end{array}\right)
$$

which reduces to the set of equations

$$
\begin{aligned}
{\left[(m a+4)^{2} \mathbb{1}-\frac{1}{4} \not D_{e o} \not D_{o e}\right] \boldsymbol{\psi}_{e} } & =(m a+4) \boldsymbol{b}_{e}+\frac{1}{2} \not D_{e o} \boldsymbol{b}_{o} \\
(m a+4) \boldsymbol{\psi}_{o} & =\boldsymbol{b}_{o}+\frac{1}{2} \not D_{o e} \boldsymbol{\psi}_{e}
\end{aligned}
$$

The solution to Eq. 22 can then be found by first solving the preconditioned system $M_{\text {prec }} \boldsymbol{\psi}_{e}=\boldsymbol{b}_{\text {prec }}$ with

$$
M_{\mathrm{prec}}=\left[(m a+4)^{2} \mathbb{1}-\frac{1}{4} \not D_{e o} \not D_{o e}\right] \quad \text { and } \quad \boldsymbol{b}_{\mathrm{prec}}=(m a+4) \boldsymbol{b}_{e}+\frac{1}{2} \not D_{e o} \boldsymbol{b}_{o}
$$

for $\boldsymbol{\psi}_{e}$. The odd solution and hence the full solution can then be constructed using Eq. 24.

\subsubsection{Mixed precision}

The efficiency of Krylov space inverters implemented on GPUs is limited by the memory bandwidth between the GPU main memory and processing units. As a result, single precision inverters will run roughly twice as fast as double precision inverters. However, lattice calculations often require higher accuracy than can be achieved in single precision. A good compromise is offered by mixed-precision inverters [8]. We used the defect-correction solver. To achieve the desired precision, the bulk of the calculation is carried out in an inner loop by a fast, reduced-precision inverter to a lower tolerance. The residue and solution vectors are then updated in full precision, and the inner step is repeated until the desired precision is achieved. The results presented in this paper were produced using double precision for the updating step and single precision in the inner solver. All steps were run on the GPU.

\section{Results}

Our main goal in this work was to create a single-mass inverter that performs comparably to its multi-shift counterpart on GPUs. The underlying 


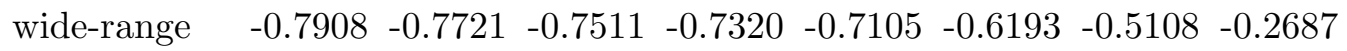

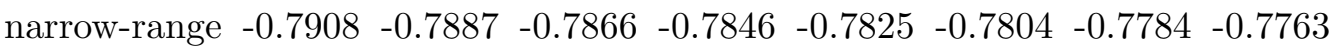

Table 2: The two sets of masses considered in this study. The values listed here are $a m$, the masses in lattice units. For the ensemble used in this study, the zero quark mass corresponds to $a m_{c}=-0.8173$. The wide-ranged set corresponds to pion masses ranging from $500 \mathrm{MeV}$ to $2200 \mathrm{MeV}$ whereas the narrow-ranged set runs from $500 \mathrm{MeV}$ to $600 \mathrm{MeV}$.

reason for this effort was the limited memory of GPUs. As will be demonstrated in the following, for the cases considered in this study, an optimized single-mass inverter not only requires less memory than its multi-shift counter part, but also outperforms it. For the single-mass and multi-shift inverters we use BiCGstab and BiCGstab-M, respectively.

In order to test the performance of the inverters, we use a small ensemble of 10 gauge configurations generated in the quenched approximation using the standard Wilson action. The lattice spacing for these configurations is $a \approx 0.1 \mathrm{fm}$. We use $24^{4}$ lattices which insures that the Wilson kernel and the sequential vector operations run at full speed (see Sections 4 and 5). Considering the number of masses in our test sets, this is also the largest lattice size that can be used with multi-shift inverters on the GPUs available to us. For larger lattices the required vectors will not fit in GPU memory. All results shown are averages taken with respect to this ensemble. The accuracy required for all solvers used in this section is $\epsilon=10^{-10}$.

We consider two sets of quark mass values: a wide-ranged set and a narrow-ranged set. The specific values can be found in Table 2. The wideranged set is taken from a typical lattice QCD study [20]. To test the effectiveness of our solver we also used a narrow-ranged set where the multi-shift inverter should have a large advantage over the single-mass one.

We start by discussing the results for the initial guessing schemes. To quantify their effectiveness, we define the baseline to be the number of iterations required to solve the multi-mass system when taking the initial guess to be the null vector. We define the gain to be

$$
\text { gain }=\frac{\text { Total } \# \text { of iters. }}{\text { baseline }}
$$

where the numerator represents the total number of iterations to solve the multi-mass system. As mention in Section 6.2, the mass values are assumed to be ordered such that $m_{1}<m_{2}<\cdots<m_{N}$, and the solutions are determined 

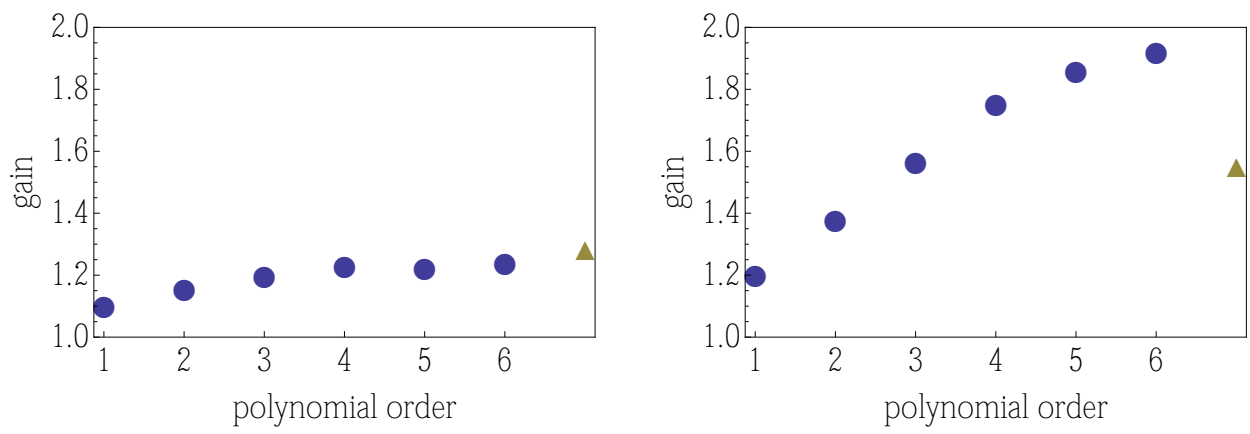

Figure 6: Results for the guessing strategies for the two sets of mass values given in Table 2: left panel is the wide-ranged set and right panel is the narrow-ranged one. Blue circles and yellow triangles correspond to polynomial extrapolation and solution space minimization, respectively.

in order starting with $m_{N}$, the heaviest mass. We choose this order since we expect the guessing procedures to improve as the number of solutions increases. Since smaller masses require more iterations, better guesses for these masses will result in a larger reduction of the total number of iterations.

We first consider the polynomial extrapolation. If the true function $\boldsymbol{\psi}(m)$ which maps mass values to their solution is slowly changing over the range of masses considered, then it should be well approximated by a finite order polynomial. It seems then sensible to use all available solutions and build the highest order polynomial to extrapolate to the new mass. However, it is possible that using too high an order might lead to oscillatory behavior (Runge's phenomenon). To investigate this, we limit the order of the polynomial used in the extrapolation and measure the gain as we increase the polynomial order. The results are shown in Fig. 6. From this figure, we see that the largest gain is achieved when using the highest possible degree polynomial. We conclude that Runge's phenomenon is not a concern and that we should use the highest polynomial order available.

The second guessing procedure considered is solution-space minimization. The results are presented in Fig. 6. We see that the solution-space minimization is either comparable or worse than polynomial extrapolation. Moreover, polynomial extrapolation is simpler to implement. We therefore use the polynomial extrapolation method as our guessing strategy. 


\begin{tabular}{llcccc}
\hline & solver & Total iters & ms/iter & total time(s) & speed-up \\
\hline \multirow{2}{*}{ narrow-range } & single-mass & 1404 & 23.8 & 33.4 & $\mathbf{2 . 1 0}$ \\
& multi-shift & 790 & 88.9 & 70.2 & \\
\hline \multirow{2}{*}{ wide-range } & single-mass & 987 & 25.7 & 25.4 & $\mathbf{2 . 2 1}$ \\
& multi-shift & 793 & 70.8 & 56.1 & \\
\hline
\end{tabular}

Table 3: Performance results for the two sets of masses. Here the speed-up is taken with regard to the multi-shift inverter.

Our single-mass inverter uses a combination of even-odd preconditioning and mixed precision. As expected, even-odd preconditioning reduces the number of iterations required for convergence by a factor of two. Similarly, using a mixed-precision solver reduces the time per iteration by a factor of two.

In the end, the important measure is the time-to-solution required by our solver. In Table 3, we compare the overall performance of the solver based on single-mass inverter to the one using a multi-shift inverter. We see that the single-mass inverter is faster than the multi-shift inverter by a factor of roughly two. While the number of iterations is larger for the single-shift inverter, the total time per iteration is much smaller. On the one hand this is due to our using mixed-precision inverters. On the other hand, the multishift inverter needs to carry out more algebra per iteration which slows it down. This slowdown is reduced in the wide-range case because the shifted solutions converge quickly and we stop updating them after few iterations.

On a related note, it is worth pointing out that using an initial guess is more effective for the narrow-ranged set. This is hardly surprising since in that case the masses are closer and the extrapolation works better. The important fact is that using an initial guess helps the single-mass inverter exactly when this is at a disadvantage with respect to the multi-shift inverter. In fact, if we didn't use an initial guess, for the narrow-ranged set the singlemass inverter would be slower than the multi-shift one, whereas for the wideranged set the single-mass inverter would still outperform it. Coupled with the slowdown experienced by the multi-shift solver in the narrow-ranged case mentioned above, using an initial guess makes the single-mass inverter perform more uniformly across different scenarios.

We conclude that our single-mass solver is not just memory efficient but it 
actually outperforms the multi-shift inverter irrespective of the distribution of quark masses considered.

\section{Conclusions}

In this study, we have addressed the issue of developing memory lean multi-mass solvers for use in lattice QCD calculations on GPUs. Such algorithms are crucial in order to carry out lattice calculations for the typical lattice sizes used today. We restricted our attention to the commonly used Krylov inverter BiCGstab and its multi-mass variant BiCGstab-M. However, we believe that our findings are applicable to other Krylov solvers such as $\mathrm{CG}$ and its multi-mass variant CG-M.

We find that a single-mass inverter using a combination of even-odd preconditioning, mixed-precision and a starting guess based on a polynomial extrapolation, is the best multi-mass solver. All three optimizations give comparable gains, each being responsible for approximately a factor of two speed-up. Thus, even though the multi-shift inverter requires significantly fewer iterations to converge, our single-mass inverter actually outperforms it by more than a factor of two. Moreover, it uses significantly less memory.

It is worth pointing out that we only considered here mixed precision inverters based on single precision. It was shown that half-precision inverters can be designed that increase performance by an additional 50\% [8]. This will make single-mass inverters outperform even more the multi-shift ones.

The motivation for our study was to develop inverters that perform well when constrained to fit in single GPU memory. Lattice QCD codes are being developed that run on multiple GPUs and the memory constraint would seem less relevant there. However, memory lean methods are also needed for multi-GPU codes due to scaling concerns. A memory lean solver requires fewer GPUs to accommodate a problem of the same size which results in a more efficient use of resources.

We conclude with a remark about the relevance of our findings for codes running on CPUs. Our conclusion should hold for all situations where the relative costs of different subroutines is the same. In the GPU case the timings are determined by the bandwidth requirements. If Wilson fermion inverters running on CPUs are bandwidth limited too, then the single-mass inverter will also perform better. 


\section{Acknowledgements}

This work is partially supported by DOE grant DE-FG02-95ER-40907. We wish to thank Mike Clark, Ron Babich and Balint Joo for useful discussions. The computational resources for this project were provided in part by the George Washington University IMPACT initiative.

\section{References}

[1] H. D. Politzer, Reliable perturbative results for strong interactions?, Phys. Rev. Lett. 30 (1973) 1346-1349.

[2] D. J. Gross, F. Wilczek, Ultraviolet behaviour of non-abelian gauge theories, Phys. Rev. Lett. 30 (1973) 1343-1346.

[3] K. G. Wilson, Confinement of Quarks, Phys. Rev. D10 (1974) 24452459 .

[4] K. G. Wilson, Quarks and Strings on a Lattice, in: A. Zichichi (Ed.), New Phenomena In Subnuclear Physics. Part A., Plenum Press, New York, 1977, p. 69.

[5] I. Montvay, G. Munster, Quantum fields on a lattice, Cambridge monographs on mathematical physics, Cambridge University Press, 1994.

[6] T. Wettig, Performance of machines for lattice QCD simulations, PoS LAT2005 (2006) 019.

[7] G. I. Egri, et al., Lattice QCD as a video game, Comput. Phys. Commun. 177 (2007) 631-639.

[8] M. A. Clark, R. Babich, K. Barros, R. C. Brower, C. Rebbi, Solving Lattice QCD systems of equations using mixed precision solvers on GPUs, Comput. Phys. Commun. 181 (2010) 1517-1528.

[9] M. A. Clark, A. D. Kennedy, Accelerating Dynamical Fermion Computations using the Rational Hybrid Monte Carlo (RHMC) Algorithm with Multiple Pseudofermion Fields, Phys. Rev. Lett. 98 (2007) 051601.

[10] A. Alexandru, A. Li, K.-F. Liu, Finite density simulations using a determinant estimator, PoS LAT2007 (2007) 167. 
[11] B. Jegerlehner, Krylov space solvers for shifted linear systems, unpublished (1996). hep-lat/9612014.

[12] R. Babich, M. A. Clark, B. Joo, Parallelizing the QUDA Library for Multi-GPU Calculations in Lattice Quantum Chromodynamics (2010).

[13] A. Alexandru, M. Lujan, C. Pelissier, B. Gamari, F. Lee, Parallel implementation of overlap fermions on gpus, in preparation (2011).

[14] H. A. van der Vorst, Bi-cgstab: A fast and smoothly converging variant of bi-cg for the solution of nonsymmetric linear systems, SIAM Journal on Scientific and Statistical Computing 13 (1992) 631-644.

[15] NVIDIA Corporation, Cuda programming guide for cuda toolkit (2010). http://developer.nvidia.com/object/gpucomputing.html.

[16] NVIDIA Corporation, Fermi compute architecture white paper (2010). http://www.nvidia.com/object/fermi_architecture.html.

[17] L. Plagne, F. Hulsemann, Improving large vector operations with $\mathrm{c}++$ expression template and atlas, in: Multiparadigm Programming with Object-Oriented Languages.

[18] J. Hoberock, N. Bell, Thrust: A parallel template library, 2010. Version 1.3.0.

[19] Y. Saad, Iterative Methods for Sparse Linear Systems, Society for Industrial and Applied Mathematics, second edition, 2003.

[20] A. Alexandru, F. X. Lee, Neutron electric polarizability, PoS LAT2009 (2009) 144. 